\title{
Genetic Markers (FASN, SCD and GH gene) and Metabolic Pathways Associated with Meat Fatty Acid Profile in Cattle (Bos taurus)
}

ISSN: 2637-7659

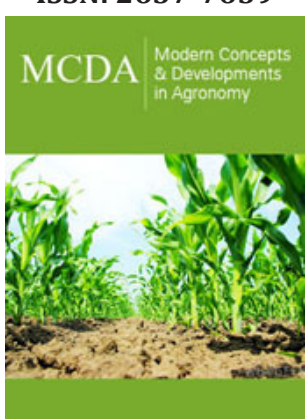

*Corresponding author: Mateja Pećina, assistant, Svetošimunska street 25, Zagreb, Croatia

\section{Submission: 侮June 15, 2020}

Published: 侮 August 11, 2020

Volume 7 - Issue 1

How to cite this article: Mateja Pećina, Nataša Hulak. Genetic Markers (FASN, SCD and GH gene) and Metabolic Pathways Associated with Meat Fatty Acid Profile in Cattle (Bos taurus). Mod Concep Dev Agrono. 7(1). MCDA. 000653. 2020. DOI: 10.31031/MCDA.2020.07.000653

Copyright@ Mateja Pećina, This article is distributed under the terms of the Creative Commons Attribution 4.0 International License, which permits unrestricted use and redistribution provided that the original author and source are credited.

\section{Mateja Pećina ${ }^{1 *}$ and Nataša Hulak ${ }^{2}$}

${ }^{1}$ Department of animal science and technology, University of Zagreb Faculty of Agriculture, Croatia

${ }^{2}$ Department of microbiology, University of Zagreb Faculty of Agriculture, Croatia

\begin{abstract}
Meat is known to man as a natural food resource product for his important energy rich and nutritious features. It is quite important in maintaining a balanced and healthy diet as well as being essential for human physiology and growth. Carbohydrates, proteins, and fat make the three major macronutrient pillars in human consumption. Fat in meat is deposited in intramuscular, intermuscular, and subcutaneous adipose stores mainly in the form of glycerol esters, phospholipids, cholesterol, and fatty acid esters. Today we know that fatty acids in ruminants consists of high amounts of saturated fatty acids (SFA) and low amounts of polyunsaturated fatty acids (PUFA), while ruminant tissues contain higher proportions of trans fatty acids. The composition of those fatty acids can be in a way influenced by environmental and genetic factors.
\end{abstract}

Keywords: Fatty acid composition; Cattle; Gene; Genetic factors

Abbreviations: FASN: Fatty Acid Synthase; SCD: Stearoyl-Coenzyme Desaturase; GH: Growth Hormone; SFA: Saturated Fatty Acids; PUFA: Polyunsaturated Fatty Acids

\section{Introduction}

Precursor of fatty acid synthesis in ruminants is the acetate which should be converted into acetyl-CoA by acetyl CoA synthetase and incorporated into fatty acids. Conversion of acetate to acetyl-CoA is performed in adipose tissue, the largest pathway to obtain the fatty acids in ruminants [1]. Many studies have attempt to identify and describe genes which play an important role in beef fatty acid metabolic pathways, as it is known that several enzymes affect lipid metabolism. One of them is the FASN (fatty acid synthase) gene found on the 19 chromosomes in cattle (Bos taurus) (NCBI Gene ID: 281152). In mitochondria, pyruvate is subsequently brought into the Krebs cycle to yield ATP. One of the by-products of this reaction is acetyl-CoA where together with malonyl-CoA becomes the substrate for FASN. FASNs gene main homework is to catalyze biosynthesis of fatty acid palmitate in a nicotinamide adenine dinucleotide phosphate-reduced (NADPH) dependent reaction [2]. Zhang et al. [3] reported that the bovine FASN gene encodes the thioesterase (TE) domain and found that the g.17924A>G SNP causes an amino change from threonine to alanine was associated. This was associated with some of the individual and grouped fatty acids in the it should be in italic muscle of American Angus cattle. Significant additive and dominance effects between the FASN SNP were detected on seven SFAs (10:0,12:0, 13:0, 14:0, 15:0, 18:0, 20:0), one branchedchain fatty acid (iso17:0), six MUFAs (monounsaturated fatty acid) (9c- 14:1, 9c- 15:1, 9c$16: 1,11 \mathrm{t}-16: 1,12 \mathrm{c}-16: 1,9 \mathrm{c}-18: 1)$ one $\mathrm{c} / \mathrm{t}$ PUFA fatty acid ( $(9 \mathrm{c}, 13 \mathrm{t} / 8 \mathrm{t}, 12 \mathrm{c}-18: 2)$ and a single PUFA (20:3n-6). The AA genotype of the FASN SNP was significantly associated with higher concentrations of the SFAs including 10:0,12:0, 13:0, 14:0 and 15:0, lower concentrations 
of the unsaturated fatty acids of 9c- 18:1 and 20:3n-6 and higher concentrations of unsaturated fatty acids including 9c- 14:1 and $12 \mathrm{c}-16: 1$ in comparison with the GG genotype $(\mathrm{P}<0.05)$ [4].

On the other hand many studies suggested SCD gene (stearoylcoenzyme A desaturase 1) found on the 26 chromosome of cattle (NCBI Gene ID: 280924) to have a major role in changing the ratio of UFA (unsaturated fatty acids) to SFA and that in the subcutaneous and intermuscular fat deposits of beef cattle majority of SFA is catalyzed to converse to MUFA, by the SCD gene. The association of SCD genotype with fatty acids composition has been investigated in Japanese black and crossbred cattle [5]. Taniguchi et al. [5] were able to identify a single nucleotide polymorphism $(\mathrm{C} / \mathrm{T})$ in the 5 th exon of bovine SCD gene (c.878C $>\mathrm{T}$ ) that causes an amino acid change from alanine to valine and found that the SNP was associated with the percentage of MUFA and the melting point of intermuscular fat in Japanese Black steers. The CC genotype of the SCD SNP was initially reported to be associated with higher amount of MUFA that included 9c-14:1, 9c-16:1 and 9c-18:1 and lower melting point in the intermuscular fat of Japanese Black Cattle [5].

On the other hand, Growth hormone gene (GH) found on the $19^{\text {th }}$ chromosome of beef cattle (Bos taurus) (NCBI Gene ID: 280804) is a hormone synthesized and secreted by somatotroph cells and it is a major regulator of postnatal growth and metabolism in mammals having an important role in lactation, protein, lipid and carbohydrate metabolism, tissue growth and fertility in cattle [6]. Ardiyanti et al. [7] made an important discovery regarding GH gene. They reported that fatty acid composition in adipose tissues in heifers had significant effects on increasing contents of fatty acid C18:1 (oleic acid), MUFA and UFA. It also had a reduced content of C16:0 (palmitic acid) and SFA. The nucleotide substitution at codons 127 and 172 of the GH gene have been significantly associated with C18:1, MUFA, SFA, and the MUFA/SFA and USFA/ SFA ratios in Japanese black cattle [7]. These results suggested that although effects of the GH gene were invisible on carcass traits it has stronger effects on fatty acid composition.

\section{Conclusion}

Fat contains essential fatty acids present in the food and provide more energy than carbohydrates and proteins. One of the biggest roles that these fatty acids have is assisting in transport and absorption of vitamins A.D.E. and $\mathrm{K}$ which are fat soluble, it provides also flavor to food and has a major role in the immune response. Fat consumption that has good fatty acid quality has been reported as essential when reduction of intake of carbohydrates food is concern. Fatty acid composition of adipose tissue depends on numerous factors such as species, age, breed, genetics, and food. They have direct impact on meat quality. When searching for healthy food trend appeared, many are looking for quality aspects in nutrition such as concentration of many "good" fatty acids, like CLA, the value of PUFA:SFA, omega acids (n6:n3) in order to try to prevent or delay the development of cardiovascular diseases or some types of tumors and diabetes [8]. Nevertheless, fat deposition along with fatty acid profiles have great influence on meat quality and the reported genes mentioned in this review show the great potential of good meat and fatty acid profile quality should be further acknowledged and observed.

\section{References}

1. Polizel Neto A, Branco RH, Bonilha SFM, Gomes HFB, Corvino TLS (2008) Functions of volatiles fatty acids on intramuscular fat deposition-Review.

2. Suzanne FJ, Jeffrey RI (2015) Molecular Pathways: Fatty Acid Synthase. Clinical Cancer Research 21(24): 5434-5438.

3. Zhang S, Knight TJ, Reecy JM, Beitz DC (2008) DNA polymorphisms in bovine fatty acid synthase are associated with beef fatty acid composition. Animal Genetics 39(1): 62-70.

4. Li C, Aldai N, Vinsky M, Dugan MER, McAllister TA (2011) Association analyses of single nucleotide polymorphisms in bovine stearoyl-CoA desaturase and fatty acid synthase genes with fatty acid composition in commercial cross-bred beef steers. Stichting International Foundation for Animal Genetics 43(1): 93-97.

5. Taniguchi M, Utsugi T, Oyama K, Mannen H, Kobayashi M, et al. (2004) Genotype of stearoyl-CoA desaturase is associated with fatty acid composition in Japanese Black cattle. Mammalian Genome 15(2): 142148.

6. Lucy MC (2008) Functional differences in the growth hormone and insulin-like growth factor axis in cattle and pigs: implications for postpartum nutrition and reproduction. Reproduction in Domestic Animals 43(Suppl 2): 31-39.

7. Ardiyanti A, Oki Y, Suda Y, Suzuki K, Chikuni K, et al. (2009) Effects of GH gene polymorphism and sex on carcass traits and fatty acid compositions in Japanese Black cattle. Animal Science Journal 80(1): 62-69.

8. Silva Martines da T, Antunes de Lemos MV, Muller LF, Baldi F, Rodrigues de Amorin T, Ferrinho AM, et al. (2018) Fat deposition, fatty acid composition and it's relationship with meat quality and human health. Meat Acience and Nutrition. 